\title{
Fiabilidad del dispositivo CW-NIRS humon beta para la medición de saturación de oxígeno muscular durante el esquí alpino: un estudio piloto
}

\author{
Reliability of the CW-NIRS humon beta device for measuring muscle oxygen saturation during alpine \\ skiing: a pilot study
}

\begin{abstract}
Miguel Fuentes Lozano; Pablo J. Gómez-López ${ }^{1}$
${ }^{1}$ Departamento Educación Física y Deportiva de la Facultad de Ciencias de Deporte. SkiingLab, Instituto Mixto Universitario Deporte y Salud (IMUDS), Universidad de Granada. Grupo Investigación HUM764.

https://orcid.org/0000-0001-7249-6541
\end{abstract}

Detalles del artículo:

Número de palabras:4.848; Tablas:1; Figuras: 0; Referencias:23

Recibido: agosto 2021; Aceptado: agosto 2021; Publicado: diciembre 2021

Conflicto de interés: El autor declara que no existen conflictos de interés.

Correspondencia del autor: Miguel Fuentes Lozano, miki1@ hotmail.es

\section{Resumen}

El siguiente estudio analizó la fiabilidad de un nuevo dispositivo (Humon Beta, Dynometrics, Inc. Boston), de onda continua (continuous wave, CW), evaluando la Sm02 del vasto lateral dominante durante cuatro estadios (basal, calentamiento, ejercicio, recuperación) a través de un protocolo de esquí alpino en simulador. Trece participantes sanos fueron evaluados mediante un diseño de medidas repetidas (test re-test) con 48 horas de separación entre pruebas. Los resultados muestran tendencia a la fiabilidad, absoluta y relativa para las medidas de Sm02 pico y media durante los cuatro estadios (CV: 5,14 - 12,90; ICC: 0,40-0,87). $\mathrm{La} \mathrm{SmO} 2$ es un condicionante limitador del rendimiento, por consiguiente, su posible modificación mediante el entrenamiento sería beneficiosa para el desarrollo de esta práctica deportiva. El dispositivo CW-NIRS puede ser una herramienta fiable para monitorizar los cambios de la $\mathrm{SmO} 2$, en diferentes estadios para los esquiadores de esquí alpino, dando información fisiológica relevante para la práctica.

Palabras claves: SmO2, iMUDS, espectroscopia de infrarrojo cercano, simulador de esquí.

\section{Abstract}

The following study analyzed the reliability of a new continuous wave $(\mathrm{CW})$ device (Humon Beta, Dynometrics, Inc. Boston), assessing the Sm02 of the vastus lateralis dominant during four stages (baseline, warm-up, exercise, recovery) through a simulator alpine skiing protocol. Thirteen healthy participants were evaluated using a repeated measures design (test re-test) with 48 hours between tests. Results show absolute and relative reliability, or trend reliability, for peak and mean $\mathrm{SmO} 2$ measures during the four stages (CV: 5.14 - 12.90; ICC: $0.40-0.87)$. SmO2 is a performance limiting factor, therefore, its possible modification through 
training would be beneficial for the development of this sport. The CW-NIRS device can be a reliable tool to monitor $\mathrm{SmO} 2$ changes at different stages for alpine skiers, providing relevant physiological information for the practice.

Key words: SmO2, iMUDS, near infrared spectroscopy, ski simulator.

\section{INTRODUCCIÓN}

El esquí alpino es una actividad compleja que entrelaza distintos factores, tanto internos como externos, que confluyen en el esquiador y a los que él mismo debe responder. Las demandas fisiológicas y la implicación muscular de los deportistas aumentarán simultáneamente a la fuerza de reacción del suelo, así como, los cambios en los radios de giro hacia giros cortos, el aumento de la velocidad o la pendiente provocarán a su vez un aumento de estas fuerzas reactivas del suelo (Seifert et al., 2005).

Existen una amplia gama de cualidades física y habilidades neuromusculares como el equilibrio, la velocidad y la agilidad que determinan el rendimiento de élite del esquí alpino (White \& Johnson, 1993). Por lo tanto, es importante y necesario conocer las demandas que requiere este deporte, puesto que representa una actividad única en cuanto a su fisiología e interacción con el medio desarrollándose en temperaturas por debajo del punto de congelación (Seifert et al., 2005). Las fuerzas musculares que se desarrollen en los virajes pueden llegar a producir niveles significativos de hipoxia e isquemia durante esta práctica (Szmedra et al., 2001).

El tipo de contracción y el nivel de actividad de los grupos musculares implicados durante el desarrollo de este deporte se caracteriza por fases repetidas de contracciones isométricas y excéntricas de gran fuerza con carácter isquémico, provocadas por altos niveles de presión intramuscular y reducciones en el flujo sanguíneo. Su fatiga periférica inhibirá el carácter contráctil y la fatiga central reducirá la producción de fuerza repercutiendo sobre las pautas de control motor (Ferguson, 2010).

A pesar de que no se obtiene información específica sobre los músculos implicados, las mediciones para la prescripción del entrenamiento deportivo se han basado tradicionalmente en la frecuencia cardiaca, las concentraciones de lactato sanguíneas o la absorción máxima de oxígeno (Farzam et al., 2018). Mejoras en el sistema oxidativo del cuerpo generan un mayor rendimiento en los eventos siendo el deterioro del consumo de oxígeno y/o el oxígeno deliberado una limitación que somete a déficits funcionales (Hamaoka et al., 2011).

La oximetría muscular de espectroscopia en el infrarrojo cercano (NIRS) proporciona de manera no invasiva información sobre los cambios en la oxigenación y hemodinámica del tejido muscular (McCully \& Hamaoka, 2000). Consecuentemente el interés por examinar la saturación de oxígeno muscular ha crecido mediante el uso de esta tecnología (Farzam et al., 2018). Concentraciones de oxihemoglobina ( $\mathrm{HbO}$ ) y desoxihemoglobina ( $\mathrm{HbR}$ ) en el tejido se pueden estimar mediante el espectro de absorción medido y la $\mathrm{SmO} 2$ se calcula tomando la relación entre la $\mathrm{HbO}$ y la concentración total de hemoglobina (HbT) (Hamaoka et al., 2011). El término saturación se refiere a la disponibilidad y funcionalidad de una escala del $0 \%$ a $100 \%$ (Feldmann et al., 2019). 
Existen diferentes investigaciones de validez y fiabilidad de la Sm02 en el campo deportivo (Crum et al., 2017; Thiel et al., 2011) que emplean el uso de estas tecnologías de oximetría muscular de espectroscopia en el infrarrojo cercano (Farzam et al., 2018; Ferrari et al., 2011; Quaresima et al., 2003).

El trabajo sobre la Sm02 por parte de los deportistas es fundamental para la mejora de su rendimiento físico, (Romer et al., 2006) siempre que estos conozcan la demanda energética de su disciplina, puesto que esta variará en función del ejercicio que se desempeñe (Neary, 2004). En esta línea Szmedra et al., (2001) emplearon la tecnología NIRS determinar si la diferencia en la máxima contracción voluntaria de los esquiadores de slalom gigante (GS) y slalom (SL) tenia incidencia en la desoxigenación del tejido muscular.

El registro de estudios que empleen en el esquí alpino tecnologías NIRS es escaso a pesar de conocer el alto compromiso al que se ve sometida la $\mathrm{SmO} 2$ en aquellos periodos de entrenamiento dependientes del sistema anaeróbico (1 a 2 minutos) (White \& Wells, 2015). En el campo del deporte son conocidos algunos dispositivos NIRS como BSX (BSX Athletics, Austin, TX, EE. UU), Moxy (Fortiori Design, LLC, Hutchinson, MN, EE. UU) o Portamon (Artinis Medical System, Einsteinweg, Países Bajos). La optimización de la intensidad del ejercicio (Ferrari et al., 2011), minimización de las lesiones (Farzam et al., 2018) o autorregulación de los mecanismos cardiovasculares en respuesta al estrés (Contreras-Briceño et al., 2019) son algunos de los beneficios que nos pueden aportar estos instrumentos asequibles.

El dispositivo CW-NIRS (Humon Beta, Dynometrics, Inc. Boston) es un nuevo dispositivo pequeño, portátil, no invasivo, de un bajo precio económico. Hasta la fecha no se tiene conocimiento de la existencia de un estudio que evalúe su fiabilidad para la medición de la SmO2 durante el desarrollo del esquí alpino. Por lo tanto, el objetivo de la investigación fue determinar si los datos obtenidos del CW-NIRS para las mediciones de $\mathrm{SmO} 2$ durante el desarrollo de un protocolo de esquí alpino pueden reproducirse en ensayos idénticos posteriores por los mismos participantes. Tras emplear el método validado de la NIRS, se plantea la hipótesis de que las medidas de CW-NIRS mostrarán fuertes correlaciones y fiabilidad entre los ensayos.

\section{MATERIAL Y MÉTODOS}

\section{Diseño}

Se empleó un diseño de medidas repetidas (test re-test) para evaluar la $\mathrm{SmO} 2$ mediante un mismo protocolo de esquí en simulador. El estudio planteado tiene un diseño descriptivo según el objeto de la investigación. Cabe mencionar que se trata de un estudio prospectivo en cuanto a la direccionalidad y longitudinal en lo que se refiere al número de mediciones. Para el grado de control de la investigación, nos encontramos en un estudio no experimental. Del mismo modo encontramos el presente estudio como un estudio piloto con motivo de un tamaño pequeño de la muestra, siendo necesarias muestras superiores a 50 sujetos para estudios de fiabilidad en ciencias deportivas (Garcia-Ramos \& Janicijevic, 2020).

\section{Participantes}


El muestreo empleado fue no probabilístico por conveniencia. Cuatro de un total de 17 participantes que aceptaron formar parte del estudio no cumplimentaron los criterios de inclusión. Un total de 2 mujeres (media \pm desviación estándar; edad: 23,5 \pm 0,7 años; estatura: $157,0 \pm 1,4 \mathrm{~cm}$; masa corporal: $56,6 \pm 6,9 \mathrm{~kg}$; masa grasa corporal: $17,4 \pm 5,9 \mathrm{~kg}$ ) y 11 hombres (media \pm desviación estándar; edad: 33,0 $\pm 10,2$ años; estatura: 176,5 \pm 5,6 cm; masa corporal: $76,7 \pm 13,5 \mathrm{~kg}$; masa grasa corporal: $14,4 \pm 7,5 \mathrm{~kg}$ ) participaron voluntariamente en el estudio. Todos los participantes eran físicamente activos y sin limitaciones físicas ni lesiones musculoesqueléticas que pudieran tener repercusión en las pruebas a someterse. Todos los participantes fueron informados sobre los procedimientos a utilizar en la investigación y firmaron el consentimiento informado previo a la misma. Los criterios de inclusión fueron: (a) tener una edad entre 17-55 años, (b) no tener patologías en miembros superiores o inferiores, (c) estar sanos, (d) practicar actividad física regular y (e) presentar el consentimiento informado.

Los criterios de exclusión para esta investigación fueron: (a) no disponer de medios técnicos para esquiar en simulador mediante el viraje en cuña fluida a distintas velocidades e inclinaciones, (b) no asistir al 100\% de las mediciones planificadas y (c) realizar entrenamientos paralelos al estudio que pudieran suponer una variable contaminante. Una semana antes de la intervención se contactó con los participantes y se les facilitó el consentimiento informado que debían devolver firmado para poder participar en la presente investigación.

\section{Medidas}

Los parámetros de estatura, peso y masa corporal se recogieron con el instrumento InBody 270 (InBody® 270). La prueba se desarrolló en un simulador de esquí MaxxTracks (C) MaxxTracks Indoor Skislopes 2018) diseñado para fines de entrenamiento, investigación y docencia, con unas dimensiones de 12.50x6.50m, situado en el iMUDS (Instituto Mixto Universitario Deporte y Salud) en el Parque Tecnológico de la Salud de Granada (España).

El registro de $\mathrm{SmO} 2$ se realizó utilizando un dispositivo CW-NIRS (Humon Beta, Dynometrics, Inc. Boston), validado previamente (Farzam et al., 2018), con una frecuencia de muestreo de 1Hz. Este dispositivo se controló mediante un reloj Garmin Forerunner 235 (Garmin Forerunner®) aplicando la app Garmin Connect a través de un dispositivo móvil (Xiaomi RedmiNote8T, G-Mobi KOREA). Los esquiadores utilizaron para el desarrollo de los virajes unos esquís (Salomon, 24 HOURS MAX), unas botas (Atomic, HAWX 110) y unos bastones (Leki, de 120cm).

Para la consecución de los virajes se ha empleado una app metrónomo (Metrónomo Beats) instaurada en un dispositivo móvil (IOS Iphone SE, software 12.3, California). Posteriormente los datos se exportaron a un ordenador (Macbook Pro Retina, software macOS High Sierra. 10.13.6, California) en formato .FIT para posteriormente convertirlos en formato .CSV. Se trabajó estadísticamente con el paquete software informático SPSS (IBM SPSS 22.0; Chicago, IL, EE. UU). 


\section{Procedimiento}

Cada participante completó un protocolo de ejercicio de esquí alpino en dos ocasiones (test re-test) separadas por 48 horas. Ambos ensayos se ejecutaron a la misma hora del día. La temperatura se fijo a $\left(21,5^{\circ} \mathrm{C}\right)$ para asegurar la consistencia de las pruebas. Los sujetos se abstuvieron de realizar ejercicios en las 24 horas anteriores a cada ensayo. Todo ejercicio que se completara durante el primer registro se replicó en el segundo. Todos los participantes al estudio habían estado previamente involucrados con el desarrollo del esquí alpino en simulador, por lo tanto, estaban familiarizados con el equipo y protocolos empleados.

Previamente a la toma de datos los sujetos fueron informados de las medidas de protección contra el Covid - 19. A la llegada al laboratorio, se obtuvo la masa corporal, estatura y peso y se aplicó el dispositivo Garmin Forerunner 235 en la mano dominante. El dispositivo Humon se posicionó en el vasto lateral de la pierna dominante, a mitad entre el trocánter mayor y el epicóndilo lateral del fémur. Se seleccionó el vasto lateral como ubicación clave debido a que forma parte del grupo extensor de la rodilla.

Antes de la colocación del dispositivo, la zona se depilaba mediante una cuchilla de afeitar desechable y se limpiaba con alcohol. El dispositivo se aseguraba al lugar mediante la cinta atlética, además de fijación con cinta de carrocero si fuera necesario, de tal forma que la luz ambiental cercana no infiriese con los detectores. Su posición se registró mediante la marca con rotulador permanente y se obtuvo una fotografía para replicar la posición en la segunda toma de datos. En posición sentada del sujeto, se registraron durante cinco minutos las medidas en reposo para la frecuencia cardiaca y SmO2. Seguidamente se paró este registro para poder dar paso al protocolo experimental.

El procedimiento experimental contenía un protocolo de esquí alpino sobre un simulador MaxxTracks. Los sujetos se sometieron a un calentamiento de cinco minutos de esquí libre con la premisa de cambiar los radios del viraje empleado. El desarrollo del test consistió en ocho minutos de esquí alpino seguidos al calentamiento, con un radio de viraje medio-corto de 1,5 metros, definido mediante marcas sobre el espejo localizado frente el tapiz. La velocidad del tapiz fue $20 \mathrm{Km} / \mathrm{h}$ y la inclinación de $12^{\circ}$ para ambos test. Para el desarrollo correcto del viraje se obligó a los sujetos a sobrepasar las líneas marcadas en el espejo además de acompañar el ritmo de los virajes mediante una app metrónomo a 50 BPM (pulsos por minuto). De esta forma, se aseguró que todos los sujetos se sometieron a la misma intensidad. Para el desarrollo del test fue obligatorio emplear un viraje de dificultad mínima de cuña fluida, de tal forma que la dificultad técnica se asemejase para todos los sujetos. Además, los sujetos emplearon el mismo tipo de viraje para ambos protocolos sin capacidad de modificar la dificultad empleada en el viraje entre test.

Los resultados de $\mathrm{SmO} 2$ y FC se registraron constantemente a lo largo del ensayo. El primer minuto de test no se registró, por lo que se tomaron valores de $\mathrm{SmO} 2$ y $\mathrm{FC}$ media y pico para cada minuto, así como global (siete minutos). Al finalizar los ocho minutos de test el sujeto vuelve a posición sentada en un tiempo máximo de un minuto para su protocolización. Una vez cumplimentado ese minuto se tomaron registros de la recuperación durante cinco minutos. 


\section{Análisis estadístico}

Los datos descriptivos se presentan como media y desviación estándar. El análisis de fiabilidad se evaluó mediante el tamaño del efecto d de Cohen (ES), el error estándar de la medición (SEM), el coeficiente de variación (CV), el coeficiente de correlación intraclase (ICC) y el correspondiente $95 \%$ de los intervalos de confianza (CI). La escala usada para interpretar la magnitud de ES fue: insignificante $(<0,2)$, pequeño $(0,2-0,5)$, moderado $(0,5-0,8)$ y grande $(\geq 0,8)$ (Cohen et al., 2013). Se categorizaron, mediante el uso de una escala cualitativa, la magnitud de los valores recopilados en el estadístico ICC, de tal forma que los valores cercanos a 0,1 se consideran bajos, 0,3 moderados, 0,5 altos, 0,7 muy altos y los cercanos a 0,9 extremadamente altos (Hopkins et al., 2009). Para la realización de los análisis estadísticos se ha empleado el paquete de software informático SPSS (IBM SPSS 22.0; Chicago, IL, EE. UU). Además, los análisis de fiabilidad se efectuaron mediante una hoja de cálculo personalizada (Hopkins, 2000).

\section{RESULTADOS}

Los 13 participantes que formaron parte del presente estudio completaron todos los requerimientos de la investigación. A continuación, se presentan los resultados obtenidos en el análisis de la fiabilidad para las variables de saturación de oxígeno muscular del vasto lateral dominante de los participantes y la frecuencia cardiaca expresadas en medias y picos (tabla 1).

La fiabilidad absoluta proporcionó una repetibilidad estable para la medición de $\mathrm{SmO} 2$ en los cuatro estadios para PicoSmO2 ( $\mathrm{CV}=5,14$ basal; $\mathrm{CV}=8,03$ calentamiento; $\mathrm{CV}=9,27$ ejercicio; $\mathrm{CV}=5,80$ recuperación), así como en MediaSmO2 en los estadios basal y recuperación ( $\mathrm{CV}=5,50$ basal y $\mathrm{CV}=6,20$ recuperación) con un coeficiente de variación inferior o cercano $(<1 \%)$ al $10 \%$. La fiabilidad absoluta proporcionó indicios a una repetibilidad estable para la medición de MediaSmO2 para los estadios calentamiento $(\mathrm{CV}=11,05)$ y ejercicio $(\mathrm{CV}=12,90)$. La fiabilidad relativa para evaluar la $\mathrm{SmO} 2$ de los cuatro estadios fue moderada - extremadamente alta $(\mathrm{ICC}=0,40-0,87)$. Para los estadios calentamiento y ejercicio, la fiabilidad relativa para evaluar la Sm02 fue moderada - alta (ICC $=0,40-0,55)$ mientras que para los estadios basal y recuperación fue alta - extremadamente alta (ICC $=0,65$ $-0,87)$.

Por otro lado, la fiabilidad absoluta proporcionó una repetibilidad estable para la medición de MediaFc en los estadios calentamiento $(\mathrm{CV}=6,56)$ y recuperación $(\mathrm{CV}=9,83)$. También la repetibilidad de PicoFc fue estable en los estadios calentamiento $(\mathrm{CV}=6,51)$ y ejercicio $(\mathrm{CV}=6,90)$. La fiabilidad absoluta dio indicios a una repetibilidad estable para la medición MediaFc en los estadios basal $(\mathrm{CV}=11,77)$ y ejercicio $(\mathrm{CV}=12,08)$, y PicoFc en los estadios basal $(C V=13,58)$ y recuperación $(C V=14,44)$. La fiabilidad relativa para evaluar la Fc fue moderada - alta para el estadio basal $(\mathrm{ICC}=0,28-0,55)$, muy alta para el estadio calentamiento $(\mathrm{ICC}=0,77-0,79)$, alta - extremadamente alta para el estadio ejercicio $(\mathrm{ICC}=$ $0,67-0,85)$. 
Fuentes Lozano, M., Gómez-López, P.J. (2021). Fiabilidad del dispositivo

CW-NIRS Humon Beta para la medición de saturación de

oxígeno muscular durante el esquí alpino: un estudio

piloto. Journal of Physical Education

and Human Movement, 3(2), 7-16.

Tabla 1. Resultados del análisis de fiabilidad para las variables $\mathrm{SmO} 2$ y HR

\begin{tabular}{|c|c|c|c|c|c|c|c|}
\hline \multirow[t]{2}{*}{ Variables } & \multicolumn{4}{|c|}{ Participantes $(n=13)$} & \multicolumn{3}{|c|}{ Vasto lateral } \\
\hline & Fases & Test & Re test & ES & SEM & CV $(95 \% \mathrm{CI})$ & ICC (90\% CI) \\
\hline \multicolumn{8}{|c|}{ MediaSmO2 } \\
\hline & Basal & $65,6(9,5)$ & $66,1(7,9)$ & 0,06 & 13,28 & $5,50(3,94 ; 9,08)$ & $0,85(0,59 ; 0,95)$ \\
\hline & Calentamiento & $57,6(10,3)$ & $59,3(6,3)$ & 0,2 & 14,62 & $11,05(7,92 ; 18,24)$ & $0,46(-0,09 ; 0,80)$ \\
\hline & Ejercicio & $56,5(12,3)$ & $60,8(5,7)$ & 0,45 & 16,28 & $12,90(9,25 ; 21,29)$ & $0,40(0,16 ; 0,77)$ \\
\hline & Recuperación & $66,6(7,1)$ & $65,7(6,1)$ & $-0,13$ & 9,98 & $6,20(4,45 ; 10,24)$ & $0,65(0,18 ; 0,88)$ \\
\hline \multicolumn{8}{|l|}{ MediaFc } \\
\hline & Basal & $79,8(11,5)$ & $80(15,1)$ & 0,01 & 16,79 & $11,77(8,44 ; 19,44)$ & $0,55(0,02-0,84)$ \\
\hline & Calentamiento & $116,5(13,3)$ & $111,4(15,3)$ & $-0,35$ & 12,58 & $6,56(4,64 ; 11,13)$ & $0,77(0,37 ; 0,93)$ \\
\hline & Ejercicio & $128,4(28,8)$ & $132,2(21,9)$ & 0,15 & 19,66 & $12,08(8,55 ; 20,50)$ & $0,67(0,18 ; 0,89)$ \\
\hline & Recuperación & $93,4(11,5)$ & $89,6(11,9)$ & $-0,33$ & 12,79 & $9,83(6,96 ; 16,69)$ & $0,45(-0,14 ; 0,80)$ \\
\hline \multicolumn{8}{|l|}{ PicoSmO2 } \\
\hline & Basal & $67,7(9,4)$ & $67,6(8,1)$ & 0,01 & 12,94 & $5,14(3,68 ; 8,48)$ & $0,87(0,62 ; 0,96)$ \\
\hline & Calentamiento & $61,8(8,8)$ & $63,5(5,1)$ & 0,23 & 11,48 & $8,03(5,76 ; 13,26)$ & $0,55(0,02 ; 0,84)$ \\
\hline & Ejercicio & $60,7(10,1)$ & $63,0(5,1)$ & 0,29 & 12,99 & $9,27(6,64 ; 15,29)$ & $0,53(0,01 ; 0,83)$ \\
\hline & Recuperación & $69,1(6,5)$ & $67,5(6,0)$ & $-0,25$ & 9,15 & $5,80(4,07 ; 9,37)$ & $0,65(0,18 ; 0,88)$ \\
\hline \multicolumn{8}{|l|}{$\mathrm{PicoFc}$} \\
\hline & Basal & $91,9(14,1)$ & $97,2(15,6)$ & 0,35 & 15,72 & $13,58(9,74 ; 22,42)$ & $0,28(-0,30 ; 0,71)$ \\
\hline & Calentamiento & $134,0(15,1)$ & $128,4(19,2)$ & $-0,32$ & 13,16 & $6,51(4,61 ; 11,06)$ & $0,79(0,42 ; 0,94)$ \\
\hline & Ejercicio & $143,8(22,9)$ & $146,4(24,2)$ & 0,11 & 16,21 & $6,90(4,89 ; 11,71)$ & $0,85(0,56 ; 0,95)$ \\
\hline & Recuperación & $118,3(18,0)$ & $117,3(13,7)$ & $-0,06$ & 13,60 & $14,44(10,23 ; 24,52)$ & $-0,14(-0,64 ; 0,45)$ \\
\hline
\end{tabular}

Abreviaturas: MediaSmO2, Media de saturación de oxígeno muscular; MediaFC, Media de frecuencia cardiaca; PicoSmO2, Pico de saturación de oxígeno muscula; PicoFC, Pico de frecuencia cardiaca; ES, Tamaño del Efecto; SEM, Error Estándar de la Medición; CV:

Coeficiente de Variación; ICC: Coeficiente de Correlación Intraclase; CI, 95\% Intervalos de Confianza.

\section{DISCUSIÓN}

El propósito de la presente investigación fue determinar si los datos obtenidos del CWNIRS para las mediciones de $\mathrm{SmO} 2$ durante el desarrollo de un protocolo de esquí alpino pueden reproducirse en ensayos idénticos posteriores por los mismos participantes. Los parámetros obtenidos de $\mathrm{SmO} 2$ y Fc durante los test respondieron a los cambios fisiológicos de los sujetos.

El uso de la tecnología NIRS ya se ha empleado para determinar la oxigenación muscular local en modalidades del ejercicio difícilmente replicables en laboratorios como son la escalada, la carrera en pista o el esquí (Crum et al., 2017). Esta tecnología es útil para monitorizar y evaluar la condición física de los deportistas, dando una optimización en el rendimiento deportivo de los mismos (Farzam et al., 2018). Más concretamente, en el ámbito de los deportes de invierno, encontramos trabajos de fiabilidad de este tipo de tecnologías abarcando protocolos de esquí nórdico (Cornachione et al., 2014), por consiguiente y hasta la fecha, no se tiene conocimiento de estudios de fiabilidad en materia del esquí alpino. 
Con el empleo del dispositivo CW-NIRS, para los valores del test re-test, nuestra investigación obtuvo buenos resultados para un estudio piloto. Las mediciones de $\mathrm{SmO} 2$ en cuanto a sus medias y a los picos es estable, o dan indicios de estabilidad para el diseño de medidas repetidas, obteniendo valores de fiabilidad absoluta (CV: 5,14; 12,90) cercanos o inferiores al $10 \%$. Encontramos de esta forma que las fases con mayor CV fueron las de calentamiento $(\mathrm{CV}=8,03 ; 11,05)$ y ejercicio $(\mathrm{CV}=9,27 ; 12,90)$. Esto se podría explicar a través de la observación de un incremento en los valores de Sm02 para los re test (media \pm desviación estándar) para las dos fases (tabla 1), lo que estaría directamente relacionado con una menor intensidad relativa en la segunda prueba, reflejada en la incidencia fisiológica de la $\mathrm{SmO} 2$ media y pico del vasto lateral de los deportistas. Los coeficientes de correlación intraclase tanto para MediaSmO2 como para PicoSmO2 fueron peores para las fases calentamiento y ejercicio (ICC moderados - altos) en comparación con las fases basal y recuperación (ICC altos extremadamente altos).

A razón de los resultados expuestos, se puede determinar que el dispositivo CW-NIRS puede ser una herramienta fiable para monitorizar los cambios de la $\mathrm{SmO} 2$ en diferentes estadios para los esquiadores de esquí alpino. Según la categorización de Hopkins et al., 2009, los valores que se obtuvieron en el estadístico ICC concluyen con una investigación que tiene indicios de fiabilidad y por lo tanto es exitosa.

Por otro lado, los valores obtenidos de la monitorización de la frecuencia cardiaca durante toda la prueba determinan una repetibilidad estable o con indicios de fiabilidad tanto absoluta como relativa, a pesar de, encontrar valores que superan el $10 \%$ para la fiabilidad absoluta. La confiabilidad en la utilización de un pulsómetro de muñeca para la monitorización de la frecuencia cardiaca depende de la tecnología que aplique el fabricante, así como de la utilización del individuo, y son considerados fiables para las mediciones de ritmo cardiaco en baja frecuencia o actividades de baja intensidad (Sirio, 2017). Por ello se plantea la utilización de instrumentación válida y fiable para la monitorización de la frecuencia cardiaca. La aparición de valores negativos dentro de la fiabilidad relativa para los parámetros de frecuencia cardiaca (ICC recuperación PicoSmO2= -0,14) se considera una desafortunada estimación con causalidad explicada por un tamaño de la muestra pequeño (Liljequist et al., 2019).

A pesar de obtener resultados prometedores, se encuentran grandes limitaciones a abordar para estudios posteriores. La principal limitación que se encontró a la hora de realizar el estudio fue un tamaño de la muestra pequeño debido a la dificultad técnica del deporte en el cual se desarrolla el protocolo, así como las medidas extraordinarias globales causadas por la crisis Covid - 19. La ausencia en el momento de la realización del registro de una App específica para el dispositivo CW-NIRS imposibilitó la utilización de instrumentos de registro de la frecuencia cardiaca externos al Garmin Forerunner 235. Por los motivos expuestos anteriormente se solicitan investigaciones futuras con un tamaño de la muestra no inferior a 50 sujetos para estudios de fiabilidad en ciencias deportivas (Garcia-Ramos \& Janicijevic, 2020). Para realizar una toma de datos más consistente se recomendaría el uso de dos dispositivos CWNIRS de tal forma que se puedan monitorizar la SmO2 de ambos vastos laterales, así como, el uso de tecnología para monitorizar la frecuencia cardiaca previamente validada y fiable. A su vez, se recomendaría realizar sesiones de familiarización con el protocolo puesto que la práctica de esquí alpino en simulador requiere unas condiciones físicas y técnicas excepcionales en comparación con otras prácticas deportivas y, en consecuencia, esto podría acusarse en la 
Fuentes Lozano, M., Gómez-López, P.J. (2021). Fiabilidad del dispositivo

CW-NIRS Humon Beta para la medición de saturación de

oxígeno muscular durante el esquí alpino: un estudio

piloto. Journal of Physical Education

and Human Movement, 3(2), 7-16.

primera práctica sobre el mismo. Algunas variaciones de error podrían atribuirse a una contaminación sobre la investigación, de modo que el tejido adiposo interfiere en la penetración de la luz del sistema CW-NIRS y consecuentemente en los resultados (Turner et al., 2013). De cara al futuro sería importante considerar el grosor del tejido adiposo de los esquiadores.

\section{CONCLUSIONES}

El presente estudio piloto de fiabilidad del dispositivo NIRS de espectroscopia de infrarrojo cercano (Humon Beta, Dynometrics, Inc. Boston) (1) es fiable o tiene tendencia a una fiabilidad para la medición de la saturación de oxígeno muscular del vasto lateral de los participantes sanos que participaron en un protocolo de cuatro estadios de esquí alpino. Se evaluó su uso mediante un diseño de medidas repetidas (test re-test) de esta tecnología portátil, económica, no invasiva y de fácil adquisición. Desde la perspectiva del estudio de los condicionantes limitadores del rendimiento físico del esquí alpino, la $\mathrm{SmO} 2$ sería uno de ellos y por consiguiente su posible modificación mediante el entrenamiento sería beneficiosa para el desarrollo de esta práctica deportiva. La monitorización en tiempo real, así como, su condición no invasiva podría ser un aspecto diferencial con respecto a otros dispositivos para el control del rendimiento de los esquiadores. Esta investigación obtuvo buenos resultados para un estudio piloto, pero se requiere de nuevos trabajos para un futuro.

\section{Referencias}

Cohen, G., Gull, E., Reichman, D. R., Millis, A. J., \& Rabani, E. (2013). Numerically exact long-time magnetization dynamics at the nonequilibrium Kondo crossover of the Anderson impurity model. Physical Review B Condensed Matter and Materials Physics, 87(19). https://doi.org/10.1103/PhysRevB.87.195108

Contreras-Briceño, F., Espinosa-Ramirez, M., Hevia, G., Llambias, D., Carrasco, M., Cerda, F., López-Fuenzalida, A., García, P., Gabrielli, L., \& Viscor, G. (2019). Reliability of NIRS portable device for measuring intercostal muscles oxygenation during exercise. Journal of Sports Sciences, 37(23), 2653-2659. https://doi.org/10.1080/02640414.2019.1653422

Crum, E. M., O'connor, W. J., Van Loo, L., Valckx, M., \& Stannard, S. R. (2017). Validity and reliability of the Moxy oxygen monitor during incremental cycling exercise. European Journal of Sport Science, 17(8), 1037-1043. https://doi.org/10.1080/17461391.2017.1330899

Farzam, P., Starkweather, Z., \& Franceschini, M. A. (2018). Validation of a novel wearable, wireless technology to estimate oxygen levels and lactate threshold power in the exercising muscle. Physiological Reports, 6(7), 1-14. https://doi.org/10.14814/phy2.13664

Feldmann, A., Schmitz, R., \& Erlacher, D. (2019). Near-infrared spectroscopy-derived muscle oxygen saturation on a $0 \%$ to $100 \%$ scale: reliability and validity of the Moxy Monitor. Journal of Biomedical Optics, 24(11), 1. https://doi.org/10.1117/1.jbo.24.11.115001

Ferguson, R. A. (2010). Limitations to performance during alpine skiing. Experimental Physiology, 95(3), 404-410. https://doi.org/10.1113/expphysiol.2009.047563

Ferrari, M., Muthalib, M., \& Quaresima, V. (2011). The use of near-infrared spectroscopy in understanding skeletal muscle physiology: Recent developments. Philosophical Transactions of the Royal Society, 369(1955), 45774590. https://doi.org/10.1098/rsta.2011.0230

Garcia-Ramos, A., \& Janicijevic, D. (2020). Potential benefits of multicenter reliability studies in sports science: A practical guide for its implementation. Isokinetics and Exercise Science, 28(2), 199-204. https://doi.org/10.3233/IES-192242

Hamaoka, T., McCully, K. K., Niwayama, M., \& Chance, B. (2011). The use of muscle near-infrared spectroscopy in sport, health and medical sciences: Recent developments. Philosophical Transactions of the Royal Society A: Mathematical, Physical and Engineering Sciences, 369(1955), 4591-4604. https://doi.org/10.1098/rsta.2011.0298

Hopkins, W. G., Marshall, S. W., Batterham, A. M., \& Hanin, J. (2009). Progressive statistics for studies in sports medicine and exercise science. Medicine and Science in Sports and Exercise, 41(1), 3-12. https://doi.org/10.1249/MSS.0b013e31818cb278 
Fuentes Lozano, M., Gómez-López, P.J. (2021). Fiabilidad del dispositivo

CW-NIRS Humon Beta para la medición de saturación de

oxígeno muscular durante el esquí alpino: un estudio

piloto. Journal of Physical Education

and Human Movement, 3(2), 7-16.

Liljequist, D., Elfving, B., \& Roaldsen, K. S. (2019). Intraclass correlation - A discussion and demonstration of basic features. In PLoS ONE (Vol. 14, Issue 7). https://doi.org/10.1371/journal.pone.0219854

McCully, K. K., \& Hamaoka, T. (2000). Near_Infrared_Spectroscopy_What_Can_It_Tell_Us.6.pdf. https://doi.org/McCully,

K. K., \& Hamaoka, T. (2000). Near-infrared spectroscopy: what can it tell us about oxygen saturation in skeletal muscle?. Exercise and sport sciences reviews, 28(3), 123-127.

Neary, J. P. (2004). Application of near infrared spectroscopy to exercise sports science. Canadian journal of applied physiology, 29(4), 488-503.

Quaresima, V., Lepanto, R., \& Ferrari, M. (2003). The use of near infrared spectroscopy in sports medicine. Journal of Sports Medicine and Physical Fitness, 43(1), 1-13.

Romer, L. M., Lovering, A. T., Haverkamp, H. C., Pegelow, D. F., \& Dempsey, J. A. (2006). Effect of inspiratory muscle work on peripheral fatigue of locomotor muscles in healthy humans. Journal of Physiology, 571(2), 425-439. https://doi.org/10.1113/jphysiol.2005.099697

Seifert, J. G., Kipp, R. W., Amann, M., \& Gazal, O. (2005). Muscle damage, fluid ingestion, and energy supplementation during recreational alpine skiing. International Journal of Sport Nutrition and Exercise Metabolism, 15(5), 528536. https://doi.org/10.1123/ijsnem.15.5.528

Sirio, L. (2017). Confiabilidad de parámetros fisiológicos estimados por elementos vestibles (wearables). Ritmo cardíaco, posición y aceleración, ECG. 5 . XVI Seminario de Ingeniería Biomédica, Universidad de la República, Uruguay.

Szmedra, L., Im, J., Nioka, S., Chance, B., \& Rundell, K. W. (2001). Hemoglobin/myoglobin oxygen desaturation during Alpine skiing. Medicine and Science in Sports and Exercise, 33(2), 232-236. https://doi.org/10.1097/00005768200102000-00010

Thiel, C., Vogt, L., Himmelreich, H., Hübscher, M., \& Banzer, W. (2011). Reproducibility of muscle oxygen saturation. International Journal of Sports Medicine, 32(4), 277-280. https://doi.org/10.1055/s-0030-1269922

Turner, L. A., Tecklenburg-Lund, S., Chapman, R. F., Stager, J. M., Duke, J. W., \& Mickleborough, T. D. (2013). Inspiratory loading and limb locomotor and respiratory muscle deoxygenation during cycling exercise. Respiratory Physiology and Neurobiology, 185(3), 506-514. https://doi.org/10.1016/j.resp.2012.11.018

White, G. E., \& Wells, G. D. (2015). The effect of on-hill active recovery performed between runs on blood lactate concentration and fatigue in alpine ski racers. The Journal of Strength \& Conditioning Research, 29(3), 800-806.

White, A. T., \& Johnson, S. C. (1993). Physiological Aspects and Injury in Elite Alpine Skiers. Sports Medicine: Evaluations of Research in Exercise Science and Sports Medicine, 15(3), 170-178. https://doi.org/10.2165/00007256-199315030-00003 